\title{
High-Resolution Melting Analysis to Detect Antimicrobial Resistance Determinants in South African Neisseria gonorrhoeae Clinical Isolates and Specimens
}

\author{
Nireshni Mitchev $\mathbb{D}^{1},{ }^{1}$ Ravesh Singh $\left(\mathbb{D},{ }^{1,2}\right.$ Veron Ramsuran ${ }^{(D)},{ }^{1}$ Arshad Ismail ${ }^{(D)}{ }^{3}$ \\ Mushal Allam $\left(\mathbb{0},{ }^{3,4}\right.$ Stanford Kwenda $\left(\mathbb{D},{ }^{3}\right.$ Florah Mnyameni, ${ }^{3}$ Nigel Garrett $₫$, 5,6 \\ Khine Swe Swe-Han $\oplus^{1,2}$ Abraham J. Niehaus $₫{ }^{1}{ }^{1}$ and Koleka P. Mlisana $\oplus^{1,5,7}$ \\ ${ }^{1}$ School of Laboratory Medicine and Medical Sciences, University of KwaZulu Natal (UKZN), Durban, South Africa \\ ${ }^{2}$ National Health Laboratory Service, Durban, South Africa \\ ${ }^{3}$ Sequencing Core Facility, National Institute for Communicable Diseases, National Health Laboratory Service, \\ Johannesburg, South Africa \\ ${ }^{4}$ Department of Genetics and Genomics, College of Medicine and Health Sciences, United Arab Emirates University, Al Ain, UAE \\ ${ }^{5}$ Centre for the AIDS Programme of Research in South Africa, Durban, South Africa \\ ${ }^{6}$ School of Nursing and Public Health, UKZN, Durban, South Africa \\ ${ }^{7}$ National Health Laboratory Service, Johannesburg, South Africa
}

Correspondence should be addressed to Nireshni Mitchev; nireshninaidoo@live.co.za

Received 23 October 2021; Accepted 6 January 2022; Published 18 January 2022

Academic Editor: Faham Khamesipour

Copyright ( 2022 Nireshni Mitchev et al. This is an open access article distributed under the Creative Commons Attribution License, which permits unrestricted use, distribution, and reproduction in any medium, provided the original work is properly cited.

Background. Antimicrobial resistance is limiting treatment options for Neisseria gonorrhoeae infections. To aid or replace culture and the syndromic management approach, molecular assays are required for antimicrobial susceptibility testing to guide appropriate and rapid treatment. Objective. We aimed to detect single-nucleotide polymorphisms and plasmids associated with antimicrobial resistance from $N$. gonorrhoeae isolates from a clinic population in South Africa, using real-time PCR as a rapid test for AMR detection. Methods. N. gonorrhoeae isolates, from female and male patients presenting for care at a sexually transmitted infections clinic in Durban, South Africa, were analysed using phenotypic and genotypic methods for identification and antibiotic susceptibility testing (AST). Real-time PCR and high-resolution melting analysis were used to detect porA pseudogene (speciesspecific marker) and resistance-associated targets. Whole-genome sequencing was used as the gold standard for the presence of point mutations. Results. The real-time porA pseudogene assay identified all N. gonorrhoeae-positive isolates and specimens. Concordance between molecular detection (real-time PCR and HRM) and resistance phenotype was $\geq 92 \%$ for $b l a_{\mathrm{TEM}}$ (HLR penicillin), rpsJ_V57M (tetracycline), tetM (tetracycline), and gyrA_S91F (ciprofloxacin). Resistance determinants 16SrRNA_C1192U (spectinomycin), mtrR_G45D (azithromycin), and penA_D545S, penA_mosaic (cefixime/ceftriaxone) correlated with the WHO control isolates. Conclusions. Eight resistance-associated targets correlated with phenotypic culture results. The porA pseudogene reliably detected $N$. gonorrhoeae. Larger cohorts are required to validate the utility of these targets as a convenient culture-free diagnostic tool, to guide STI management in a South African population.

\section{Introduction}

Sexually transmitted infections (STIs) are a global public health concern, with an annual estimate of 87 million new cases of Neisseria gonorrhoeae infection [1]. Globally, N. gonorrhoeae has developed resistance to most antibiotics, including third- generation cephalosporins, cefixime and ceftriaxone. The World Health Organization (WHO) call to end the STI epidemic as a public health concern emphasizes a need to strengthen technologies and improve knowledge on the prevalence, resistance patterns, and public health interventions to combat STIs, especially in low- and middle-income countries [2]. 
For N. gonorrhoeae, the sensitivity of microscopy for diagnosis is $90-95 \%$ in men and $50-70 \%$ for presumptive diagnosis in women [3]. The sensitivity of culture ranges from $85-95 \%$ in patients with recent and symptomatic infection to as low as $50 \%$ in asymptomatic patients $[4,5]$. A challenge to STI management is delayed or ineffective treatment initiation whilst awaiting laboratory diagnosis (48-72 hours), which could lead to patient loss to followup, $[6,7]$ and increased risk of infection transmission to sexual partners. In well-resourced settings, sensitive and rapid nucleic acid amplification tests (NAATs) have largely replaced phenotypic identification of N. gonorrhoeae [5]. Although NAATs are effective in diagnosis at POC, most settings treat patients empirically at the first visit.

Syndromic and empiric treatment contributes to the development of resistance to currently recommended drugs in many parts of the world [8-10]. N. gonorrhoeae mechanisms of resistance have been well documented [11-13]. These include plasmid-mediated resistance to penicillin $\left(b l a_{\mathrm{TEM}}\right)$ and tetracycline $($ tet $M)$ and chromosomally mediated resistance to penicillin, tetracycline, spectinomycin, fluoroquinolones, azithromycin and cephalosporins [11]. Resistance to penicillin and extended-spectrum cephalosporins has been associated with modifications and recombination within pen $A$, por $B$, and ponA [14]. The mutation $L 421 \mathrm{P}$ in ponA reduces the rate of acylation with penicillin [15]. Mutations in $m t r R$, as well as its promoter region, can cause overexpression of the mtrCDE efflux pump which has been associated with resistance to penicillin, tetracycline, cefixime, ceftriaxone, and azithromycin [16, 17]. Mutations in porB which encode porinB, reduces the porin permeability. The pilQ gene encodes for pore formation in the outer membrane, mutations in which result in reduced antibiotic influx $[11,13,18,19]$. Resistance to tetracycline has been associated with mutations in $r p s J, m t r R$, and $\operatorname{por} B$ [16]. The rpsJ mutation V57M alters the binding site, thus reducing the binding affinity of tetracycline for the ribosome [11]. Resistance to ciprofloxacin is due to mutations in gyrA and parC [16]. High-level resistance to spectinomycin is due to the mutation $\mathrm{C} 1192 \mathrm{U}$ in $16 \mathrm{~S}$ rRNA by reducing antibiotic target affinity [20]. Resistance to azithromycin is often due to mutations in $23 \mathrm{~S}$ rRNA, namely, C2611T (low-level resistance) or A2059 (high-level resistance) $[11,20]$.

Molecular antimicrobial resistance testing is imperative for the control of $N$. gonorrhoeae, to facilitate antibiotic stewardship, to expedite appropriate treatment of patients, and to conserve the effectiveness of the current treatment regimen [21]. A recent study projected that continued empiric treatment without antimicrobial susceptibility testing at the POC would result in $>5 \%$ of $N$. gonorrhoeae isolates being resistant to both azithromycin and ceftriaxone within 15 years, but the use of a molecular assay could delay the emergence of resistance by 5 years $[22,23]$. Currently, the only commercially available genotypic resistance testing assay for N. gonorrhoeae is from SpeeDX (Australia), which determines resistance to ciprofloxacin by detecting the
gyrA_S91F mutation. However, due to the high prevalence of resistance to ciprofloxacin in South Africa and other African countries [21], additional assays to detect genotypic resistance to other drugs are necessary.

Whole-genome sequencing and a variety of bioinformatics tools are widely used to predict AMR and identify novel mutations in N. gonorrhoeae. These tools include Resistance Gene Identifier (RGI), Basic Local Alignment Search Tool (BLAST), Rapid Annotation Using Subsystems Technology (RAST), Antibiotic Resistance Gene-ANNOTation (ARGANNOT), ResFinder, ARIBA, ABRicate, ResFinder, PointFinder, and NG-STAR [24]. However, the cost of this technology is high, especially in low-middle-income-countries (LMIC).

Real-time polymerase chain reaction (real-time PCR) combines amplification and detection of gene targets in a single assay. Advantages include rapid detection, the ability to be implemented in high throughput settings [25], and cost effectiveness [26]. The porA pseudogene is a N. gonorrhoeae species-specific marker [27-29] and is highly conserved across a diverse range of $N$. gonorrhoeae strains making it a useful target for identification [27, 28, 30, 31].

High-resolution melt (HRM) analysis is a post-PCR analysis method, which amplifies gene targets in the presence of a fluorescent reporter dye; the increasing temperature gradient denatures the DNA and releases the dye, which then undergoes a conformational change that reduces the fluorescence. An HRM instrument records the fluorescence fluctuation and produces a melt curve of the target gene, which can be used to compare the similarity of PCR products [32]. HRM is so sensitive that it can detect a singlenucleotide polymorphism (SNP) [30]. This technology is rapid and cost effective [33], a cheaper alternative to sequencing [32, 34], and can be employed to detect SNPs associated with antibiotic resistance $[30,35,36]$.

Developing and evaluating new technologies for AMR prediction directly from patient specimens, to be used at the POC or near-patient, is currently a priority to conserve current and future antimicrobials [18]. Some resistance determinants may work on their own in increasing resistance in particular drugs. However, in some cases, a single determinant may only alter the minimum inhibitory concentration (MIC) slightly or not at all [11]. In our study, we correlated known AMR determinants with phenotypic AST (gold standard) data from our local isolates to determine which mutations in our population result in a resistant phenotype. Using real-time PCR and HRM technologies, we detected genes and mutations associated with antimicrobial resistance to penicillin, tetracycline, spectinomycin, ciprofloxacin, azithromycin, ceftriaxone, and cefixime in our local population.

\section{Materials and Methods}

2.1. Study Population and Sample Collection. Genital samples were collected from symptomatic male and female patients attending a large urban STI clinic in Durban, South Africa, as part of two clinical studies. Female patients (May 2016 -January 2017), aged 18-40 years, consented to vaginal swab 
collection (Eswab ${ }^{\circledR}$, Copan, Brescia, Italy), as reported previously [37], and male patients (June-August 2015), aged 19-60 years, consented to urethral Eswab ${ }^{\circledR}$ collection. A total of $22 \mathrm{~N}$. gonorrhoeae-positive specimens were included in this study. Ethics was approved for this study by the Biomedical Research Ethics Committee of the University of KwaZulu-Natal, BREC97/2019.

\subsection{Isolation and Identification of Neisseria gonorrhoeae.} Phenotypic identification included bright-field (Gram stain) microscopy and culture. Swabs were inoculated onto New York City and chocolate agar media and incubated for 24-48 hours at $37^{\circ} \mathrm{C}$ in a $5 \% \quad \mathrm{CO}_{2}$ incubator. Suspected $N$. gonorrhoeae colonies were confirmed using the Rapid Oxidase test and Phadebact ${ }^{\circledR}$ Monoclonal GC test (Pharmacia, Sweden). STI screening, directly from patient specimens, was performed using NAATs, Anyplex ${ }^{\mathrm{TM}}$ II STI-7 Detection (Seegene, Seoul, Korea), and Xpert ${ }^{\circledR}$ (Cepheid, CA, USA). Isolates were stored in the laboratory repository for future phenotypic and genotypic analysis.

2.3. Antimicrobial Susceptibility Testing. N. gonorrhoeae isolates from vaginal and urethral specimens were revived on nonselective Thayer Martin (antibiotic supplement excluded) and chocolate agar media for $18-24$ hours in a $37^{\circ} \mathrm{C}$ $5 \% \mathrm{CO}_{2}$ incubator. WHO N. gonorrhoeae control strains (F, G, K, L, M, N, O, and P) [38] and ATCC strain 49226 were used in this study. AST using Etest ${ }^{\circledR}$ (bioMérieux, Marcy l'Etoile, France) was performed for penicillin, tetracycline, spectinomycin, ciprofloxacin, azithromycin, ceftriaxone, and cefixime, as per the manufacturer's guidelines, using GC agar base medium supplemented with $1 \%$ Vitox $[39,40]$. The European Committee on Antimicrobial Susceptibility Testing (EUCAST) guidelines [41] were used to interpret MICs.

2.4. DNA Extraction. Genomic DNA was isolated from N. gonorrhoeae isolates and control strains (WHO F, L, O, G, $\mathrm{M}, \mathrm{P}, \mathrm{N}$, and K) [38] using the Quick-DNA ${ }^{\mathrm{TM}}$ Miniprep Plus Kit (Zymo Research, CA, USA) as per the manufacturer's instruction. DNA was extracted from patient specimens as follows: swabs were vortexed for $30 \mathrm{~s}$ while inside their Eswab $^{\circledR}$ transport tubes, whereafter $200 \mu \mathrm{L}$ of the suspension was added to a $1.5 \mathrm{~mL}$ Eppendorf tube, and the QuickDNA $^{\mathrm{TM}}$ Miniprep Plus Kit (Zymo Research, CA, USA) was used as per the manufacturer's instruction.

2.5. Real-Time PCR for porA, bla ${ }_{T E M}$, and tetM. Real-time PCR was performed on the Quant Studio 5 (ThermoFisher, CA, USA), to detect porA for identification of $N$. gonorrhoeae and plasmids bla $a_{\mathrm{TEM}}$ and $t e t M$, which confers resistance to penicillin and tetracycline, respectively.

2.6. High-Resolution Melt Analysis. We screened penA, porB, ponA, gyrA, parC, $m$ trR, rpsJ, and 16s rRNA for the presence of SNPs associated with AMR to penicillin,
TABLE 1: Oligonucleotides used in this study.

\begin{tabular}{|c|c|}
\hline Target & Primer $5^{\prime}-3^{\prime}$ \\
\hline porA_F & F_CCGTGCGTTACGATTCCCCC \\
\hline porA_R & R_ACAGCCGGAACTGGTTTCATCTG \\
\hline bla $a_{\mathrm{TEM}} \mathrm{F}$ & F_ATAGACAGATCGCTGAGATAGGTGC \\
\hline$b l a_{\mathrm{TEM}} \mathrm{R}$ & R_AAAAGCGGTTAGAGCGGCTATTG \\
\hline tetM_F & F_CCAGCCCCGTCGTCCAAATAGTC \\
\hline tetM_R & R_GCATCAATCATTTGCTCATGTGGC \\
\hline penA_F & F_CCGTGTGATTGTGGCGGTAACC \\
\hline penA_R & R_TGCCCAAGATGTTCAGGCTGC \\
\hline G545S & F_GCCGACTGCAAACGGTTACTACA \\
\hline mosaic & F_GCCGACTGCAAACGGTTACTACG \\
\hline ponA_F & F_GAGCGGTCGATAATGAGAAAATGG \\
\hline ponA_R & R_GCATCCAGCGAAACCAAAGC \\
\hline L421P & F_GGTGGTTCAAGAGCCGTTGCC \\
\hline porB_F & F_CAACAAACAATCCTTCGTCGGCTTG \\
\hline porB_R & R_GGCAAATTCGGGAGAATCGTAGCG \\
\hline G120D & F_CAGCCCCCTGAAAAACACCGA \\
\hline G120K & F_CAGCCCCCTGAAAAACACCA \\
\hline A121G & F_GGATTCCCAAGCATTGACGTTGCC \\
\hline A121D & F_GGATTCCCAAGCATTGACGTTGT \\
\hline rpsJ_F & F_GCGTTTCAACATTTTGCGTTCTCC \\
\hline rpsJ_R & R_CATCGGTAGTTTTATCGGTCCAATCC \\
\hline V57M & F_AACATTTTGCGTTCTCCGCACA \\
\hline gyrA_F & F_AAAATAACTGGAATGCCGCCTAC \\
\hline gyrA_R & R_GAAGTTGCCCTGTCCGTCTATC \\
\hline S91F & F_TACCACCCCCACGGCGATTT \\
\hline D95A & F_CGCCATACGGACGATGGTGG \\
\hline D95G & F_CGCCATACGGACGATGGTGCC \\
\hline parC_F & F_CGTGGTCGGCGAGATTTTGG \\
\hline parC_R & R_CGAACCGAAGTTGCCGATGC \\
\hline S87R & F_TACCATCCGCACGGCGACC \\
\hline S88P & F_CATCCGCACGGCGACAGTC \\
\hline 16S rRNA_F & F_AGCCGTAACACAGGTGCTGC \\
\hline 16S rRNA_R & R_GACCATTGTATGACGTGTGAAGCC \\
\hline C1192U & F_ATAAGGGCCATGAGGACTTGACA \\
\hline mtrR_F & F_GGGTTTCATTATACATACACGATTGC \\
\hline$m t r h$ & R_GATGTCGTCGCAGATACGTTGG \\
\hline G45D & F_TTTGAAATGCCAATAGAGCGCGT \\
\hline
\end{tabular}

For SNPs, a common reverse primer was used from the chromosomal gene primer set.

tetracycline, spectinomycin, ciprofloxacin, azithromycin, cefixime, and ceftriaxone, as previously described [12]. Briefly, PCR was employed to amplify the chromosomal genes (Table 1) on the SimpliAmp instrument (Life Technologies, CA, USA), using the KAPA HiFi PCR kit (KAPA Biosystems, MA, USA) as per the manufacturer's instructions. Cycling conditions were as follows for the first-round primers: $95^{\circ} \mathrm{C}$ for 3 minutes, 30 cycles of $98^{\circ} \mathrm{C}$ for 30 seconds, $55^{\circ} \mathrm{C}$ for 30 seconds, and $72^{\circ} \mathrm{C}$ for 30 seconds a final extension reaction at $72^{\circ} \mathrm{C}$ for 7 minutes. Samples were run on a $2 \%$ agarose gel to confirm PCR amplification. The PCR product was diluted $(1: 4000)$ and used as the template for HRM assays. To detect SNPs that associate with phenotypic resistance, we used HRM technology, performed on the Quant Studio 5 (ThermoFisher, CA, USA). Each HRM reaction (20 $\mu$ l total volume) consisted of $10 \mu$ l MeltDoctor ${ }^{\mathrm{TM}}$ HRM Master Mix (Applied Biosystems, CA, USA), $1.2 \mu \mathrm{l}$ of each primer $(0.3 \mathrm{pmol} / \mu \mathrm{l})$, $1 \mu \mathrm{l}$ DNA template, and nuclease-free water. Reactions 
TABLE 2: Comparison of high-resolution melt assay and whole-genome sequencing (gold standard) for the detection of antimicrobial resistance determinants in Neisseria gonorrhoeae clinical isolates and control strains.

\begin{tabular}{|c|c|c|c|c|c|}
\hline Target & $\begin{array}{c}\text { HRM vs. WGS concordance } \\
(\%)\end{array}$ & $\begin{array}{c}\text { Sensitivity (95\% CI) } \\
(\%)\end{array}$ & $\begin{array}{c}\text { Specificity }(95 \% \mathrm{CI}) \\
(\%)\end{array}$ & PPV (95\% CI) (\%) & NPV (95\% CI) (\%) \\
\hline ponA_L421P & 66.7 & $76.2(52.8-91.8)$ & $44.4(13.7-78.8)$ & $76.2(63.0-85.8)$ & $44.4(21.7-69.7)$ \\
\hline porB_G120D & 83.3 & $40.0(5.3-85.3)$ & $92.0(74.0-99.0)$ & $50.0(15.3-84.7)$ & $88.5(78.8-94.1)$ \\
\hline porB_G120K & 96.7 & $75.0(19.4-99.4)$ & $100(86.8-100)$ & 100 & $96.3(82.7-99.3)$ \\
\hline porB_A121G & 96.7 & $75.0(19.4-99.4)$ & $100(86.8-100)$ & 100 & $96.3(82.7-99.3)$ \\
\hline porB_A121D & 70.0 & $85.7(42.1-99.6)$ & $65.2(42.7-83.6)$ & $42.9(28.4-58.6)$ & $93.8(70.5-98.6)$ \\
\hline mtrR_G45D & 93.3 & $66.7(9.4-99.2)$ & $96.3(81.03-99.9)$ & 66.7 (19.9-94.1) & $96.3(83.9-99.2)$ \\
\hline rpsJ_V57M & 93.3 & $100(87.6-100)$ & $100(15.8-100)$ & 100 & 100 \\
\hline gyrA_S91F & 100 & $100(85.2-100)$ & $100(59.0-100)$ & & \\
\hline gyrA_D95A & 96.7 & $85.7(42.1-99.6)$ & $100(85.2-100)$ & 100 & $95.8(78.9-99.3)$ \\
\hline gyrA_D95G & 66.6 & $38.5(13.9-68.4)$ & $88.2(63.6-98,5)$ & $71.4(36.5-91.6)$ & $65.2(54.1-74.9)$ \\
\hline parC_S87R & 100 & $100(2.5-100)$ & $100(88.1-100)$ & 100 & 100 \\
\hline parC_S88P & 100 & $100(15.8-100)$ & $100(87.7-100)$ & 100 & 100 \\
\hline 16S_C̄ $1192 U^{*}$ & 100 & $100(2.5-100)$ & $100(88.1-100)$ & 100 & 100 \\
\hline penA_G545S* & 100 & $100(2.5-100)$ & $100(87.7-100)$ & 100 & 100 \\
\hline penA_mosaic* & 100 & $100(2.5-100)$ & $100(88.1-100)$ & 100 & 100 \\
\hline
\end{tabular}

* Only control strains were used for these targets. All isolates in this study were susceptible to spectinomycin, azithromycin, cefixime, and ceftriaxone.

were performed on the Quant Studio 5 (ThermoFisher, CA, USA) as follows: initial denaturation at $95^{\circ} \mathrm{C}$ for $10 \mathrm{~min}$, followed by 35 cycles at $95^{\circ} \mathrm{C}$ for $15 \mathrm{~s}$ and $60^{\circ} \mathrm{C}$ for $1 \mathrm{~min}$. HRM analysis was performed as follows: an initial holding step for $1 \mathrm{~min}$ at $60^{\circ} \mathrm{C}$, followed by a slow temperature increase at a rate of $0.075^{\circ} \mathrm{C} / \mathrm{s}$ to $95^{\circ} \mathrm{C}$ with continuous fluorescence signal collection. The results were analysed using High-Resolution Melt Software v3.1 (Applied Biosystems ${ }^{\mathrm{TM}}$ ).

2.7. Whole-Genome Sequencing and Assembly. DNA was extracted from isolates using the PureLink ${ }^{\mathrm{TM}}$ Microbiome DNA Purification Kit (ThermoFisher Scientific) as per the manufacturer's instructions. Paired-end libraries were prepared using the Nextera DNA Prep kit, followed by sequencing $(2 \times 75 \mathrm{bp})$ on a NextSeq platform (Illumina, Inc., USA). Briefly, Trim Galore v0.6.2 [42] was used to filter the PE reads $(Q>30$ and length $>50 \mathrm{bp}$ ). De novo assembly and polishing of assemblies were performed using SPAdes v.3.13 [43] and Shovill v1.1.0 [44], respectively. AMR markers were identified using PointFinder [45] and confirmed using Pathogenwatch [46] and Clustal Omega [47]. Whole-genome sequence data are available in DDBJ/ENA/GenBank with the BioProject number PRJNA681740.

2.8. Statistical Analysis. The sensitivity, specificity, positive predictive value (PPV), and negative predictive value (NPV) for HRM in comparison with WGS and phenotypic AST were calculated using MedCalc calculator (MedCalc Software bvba, Ostend, Belgium). Sequencing data were used as the reference for the identification of resistance-associated genes and mutations. Phenotypic AST was used as the reference for resistance prediction.

\section{Results}

3.1. Genotypic Analysis. A total of 22 paired N. gonorrhoeae isolates and clinical specimens and eight WHO control strains were included in this study. Real-time PCR detected the porA pseudogene, to identify N. gonorrhoeae, in all $22(100 \%)$ clinical isolates and specimens, and eight control strains. We observed that the $b l a_{\text {TEM }}$ plasmid showed $100 \%$ concordance in the detection of high-level resistance to penicillin when phenotypic and genotypic results were compared. Wholegenome sequencing confirmed the presence of tet $M$ and showed $100 \%$ concordance with the phenotypic data.

N. gonorrhoeae antimicrobial target genes (penA, porB, ponA, gyrA, parC, $m t r R, r p s$ J, and 16s rRNA) were investigated for the presence of resistance-associated mutations using HRM. The performance of the HRM assay to detect the presence or absence of mutations in clinical isolates was compared against the WGS data as the gold standard (Table 2). The sensitivity ranged from $38.5 \%-100 \%$, and specificity ranged from $44.4 \%-100 \%$. Positive predictive values ranged from $42.9 \%-100 \%$, and negative predictive values ranged from $44.4 \%-100 \%$. Concordance between the two methods was $\geq 83.3 \%$ for porB_G120D, porB_G120K, porB_A121G, mtrR_G45D, rpsJ_V57M, gyrA_S91F, gyrA_D95A, parC_S87R, parCS88P, 16S_C1192U, penA_G545S, and penA_mosaic.

The ability of HRM to detect the presence or absence of these resistance-conferring mutations was further evaluated by comparing HRM with phenotypic characteristics (Table 3). For penicillin, the sensitivity ranged from $4.4 \%-70.8 \%$, specificity ranged from $33.3 \%-100 \%$, positive predictive values ranged from $33.3 \%-100 \%$, and negative predictive values ranged from $14.8 \%-25.0 \%$. For tetracycline, the sensitivity ranged from $10.3 \%-100 \%$, the specificity for mtrR_G45D was $100 \%$, positive predictive values ranged from $96.4-100 \%$, and negative predictive value for mtrR_G45D was 3.7\%. For ciprofloxacin, the sensitivity ranged from $4.4 \%-100 \%$, specificity was $100 \%$, positive predictive value was $100 \%$, and negative predictive values ranged from $24.1 \%-100 \%$. Although no resistance to spectinomycin, azithromycin, cefixime, and ceftriaxone was detected in our clinical isolates, the performance of HRM to 
TABLE 3: Performance characteristics of real-time PCR and high-resolution melt assay compared to phenotypic AST (gold standard) to predict antimicrobial resistance in Neisseria gonorrhoeae clinical isolates and control strains.

\begin{tabular}{|c|c|c|c|c|c|c|}
\hline Drug $(\mu \mathrm{g} / \mathrm{mL})$ & Target & $\begin{array}{c}\text { Genotypic assay vs. AST } \\
\text { concordance }(\%)\end{array}$ & $\begin{array}{c}\text { Sensitivity (95\% } \\
\text { CI) }(\%)\end{array}$ & $\begin{array}{c}\text { Specificity }(95 \% \\
\text { CI) }(\%)\end{array}$ & $\begin{array}{c}\text { PPV }(95 \% \mathrm{CI}) \\
(\%) \\
\end{array}$ & $\begin{array}{c}\text { NPV }(95 \% \mathrm{CI}) \\
(\%) \\
\end{array}$ \\
\hline \multirow{7}{*}{$\begin{array}{l}\text { PEN (MIC } \\
0.016-.256)\end{array}$} & ponA_L421P & 63.3 & $70.8(48.9-87.4)$ & $33.3(4.3-77.7)$ & $\begin{array}{c}81.0 \\
(69.5-88.8)\end{array}$ & $22.2(7.3-51.0)$ \\
\hline & porB_G120D & 26.7 & $12.5(2.7-32.4)$ & $83.3(35.9-99.6)$ & $\begin{array}{c}75.0 \\
(27.3-96.0)\end{array}$ & $\begin{array}{c}19.2 \\
(13.9-26.0)\end{array}$ \\
\hline & porB_G120K & 30.0 & $12.5(2.7-32.4)$ & $100(54.1-100)$ & 100 & $\begin{array}{c}22.2 \\
(19.7-25.0)\end{array}$ \\
\hline & porB_A121G & 17.2 & $4.4(0.1-22.0)$ & $66.7(22.3-95.7)$ & $33.3(5.1-82.2)$ & $15.4(9.3-24.4)$ \\
\hline & porB_A121D & 53.3 & $50.0(29.1-70.9)$ & $66.7(22.3-95.7)$ & $\begin{array}{c}85.7 \\
(64.4-95.2)\end{array}$ & $\begin{array}{c}25.0 \\
(14.3-40.0)\end{array}$ \\
\hline & mtrR_G45D & 23.3 & $11.5(2.5-30.2)$ & $100(39.8-100)$ & 100 & $\begin{array}{c}14.8 \\
(13.2-16.7)\end{array}$ \\
\hline & blaTEM & 100 & $100(81.5-100)$ & $100(73.5-100)$ & 100 & 100 \\
\hline \multirow{4}{*}{$\begin{array}{l}\text { TET (MIC } \\
0.75-32)\end{array}$} & rpsJ_V57M & 96.7 & $100(88.1-100)$ & $0(0-98)$ & $\begin{array}{c}96.7 \\
(96.7-96.7)\end{array}$ & \\
\hline & mtrR_G45D & 13.3 & $10.3(2.2-27.4)$ & $100(2.5-100)$ & 100 & $3.7(3.3-4.2)$ \\
\hline & tetM & 100 & $100(85.2-100)$ & $100(59.0-100)$ & 100 & 100 \\
\hline & gyrA_S91F & 100 & $100(85.2-100)$ & $100(59.0-100)$ & & \\
\hline \multirow{4}{*}{$\begin{array}{l}\text { CIP MIC } \\
(0.002-2)\end{array}$} & gyrA_D95A & 43.3 & $26.1(10.2-48.4)$ & $100(59.0-100)$ & 100 & $\begin{array}{c}29.2 \\
(24.4-34.4)\end{array}$ \\
\hline & gyrA_D95G & 46.7 & $30.4(13.2-52.9)$ & $100(59.0-100)$ & 100 & $\begin{array}{c}30.4 \\
(25.0-36.4)\end{array}$ \\
\hline & parC_S87R & 26.7 & $4.4(0.1-22.0)$ & $100(59.0-100)$ & 100 & $\begin{array}{c}24.1 \\
(22.6-25.8)\end{array}$ \\
\hline & parC_S88P & 30 & $8.7(1.1-28.0)$ & $100(59.0-100)$ & 100 & $\begin{array}{c}25.0 \\
(22.7-27.4)\end{array}$ \\
\hline \multirow{3}{*}{$\begin{array}{l}\text { SPT MIC range } \\
(2-31) \\
\text { AZM MIC } \\
(0.016-0.38)\end{array}$} & 16S_C1192U* & 100 & $100(2.5-100)$ & $100(88.1-100)$ & 100 & 100 \\
\hline & mtrR_G45D* & 96.7 & $100(15.8-100)$ & 96.4 (81.7-99.9) & $\begin{array}{c}66.7 \\
(22.6-93.2)\end{array}$ & 100 \\
\hline & penA_D545S* & 100 & $100(2.5-100)$ & $100(87.7-100)$ & 100 & 100 \\
\hline \multirow[t]{2}{*}{ FIX/CRO ${ }^{\#}$} & penA_mosaic* & 100 & $100(2.5-100)$ & $100(88.1-100)$ & 100 & 100 \\
\hline & mtrR_G45D* & 93.3 & $100(2.5-100)$ & $93.1(73.2-99.2)$ & $\begin{array}{c}33.3 \\
(11.6-65.6) \\
\end{array}$ & 100 \\
\hline
\end{tabular}

detect mutations in the control strains was as follows: sensitivity was $100 \%$, specificity ranged from $93.1 \%-100 \%$, positive predictive values ranged from $33.3 \%-100 \%$, and negative predictive values were $100 \%$.

HRM was used to detect chromosomal gene mutations, and RTPCR was used to detect plasmid genes. * Only control strains were used for these targets. All isolates in this study were susceptible to spectinomycin, azithromycin, cefixime, and ceftriaxone. $\mathrm{PEN}=$ penicillin, $\mathrm{TET}=$ tetracycline, $\mathrm{CIP}=$ ciprofloxacin, $\mathrm{SPT}=$ spectinomycin, $\mathrm{AZM}=$ azithrom ycin, FIX = cefixime, and CRO = ceftriaxone. ${ }^{\#}$ CFM MIC for all isolates was <0.016, and the CRO MIC range was $<0.002-0.003$.

Concordance between molecular detection (real-time PCR and HRM) and resistance phenotype was $\geq 93 \%$ for $b l a_{\text {TEM }}$ (HLR penicillin), rpsJ_V57M (tetracycline), tetM (tetracycline), gyrA_S91F (ciprofloxacin), 16SrRNA_C 1192 U (spectinomycin), mtrR_G45D (azithromycin), penA_D545S (cefixime/ceftriaxone), and penA_mosaic (cefixime/ceftriaxone).

When evaluating the HRM assay to screen the 22 isolates paired clinical specimens (vaginal/urethral swabs) for the presence or absence of resistance-conferring mutations, the concordance of HRM detection between patient isolate and specimen was $>90 \%$ for all targets (Table 4 ). These excellent concordance values between patient isolate and paired specimen suggest that the data from Tables 2 and 3 can be inferred to patient specimens.

\section{Discussion}

This study used molecular techniques to identify N. gonorrhoeae and to detect genes and mutations associated with antimicrobial resistance. AST revealed that $N$. gonorrhoeae resistance to penicillin, tetracycline, and ciprofloxacin was high in our isolates. We report that spectinomycin, cefixime, and the drugs used in the syndromic management approach, ceftriaxone and azithromycin, remain effective as all isolates were susceptible at the lowest MIC. Our study shows that the sensitivity and specificity of the molecular assays for $b l a_{\text {TEM }}$ (high-level resistance to penicillin), rpsJ_V57M (lower-level resistance to tetracycline), tet $M$ (high-level resistance to tetracycline), and gyrA_S91F (resistance to ciprofloxacin) were 100\%, with $100 \%$ concordance to phenotypic AST data of patient isolates and control strains. Head-to-head comparison of HRM data 
TABLE 4: Evaluation of high-resolution melt assay for the detection of antimicrobial resistance determinants in Neisseria gonorrhoeae clinical isolates compared to direct swab specimens.

\begin{tabular}{|c|c|c|c|c|c|}
\hline \multirow{2}{*}{ Target } & \multicolumn{2}{|c|}{ Isolates $(n=22)$} & \multicolumn{2}{|c|}{ Direct specimens $(n=22)$} & \multirow{2}{*}{ Concordance (\%) } \\
\hline & Present $n(\%)$ & Absent $n(\%)$ & Present $n(\%)$ & Absent $n(\%)$ & \\
\hline ponA_L421P & $13 / 22(59.1)$ & $9 / 22(40.9)$ & $12 / 22(54.5)$ & $10 / 22(45.5)$ & 95.5 \\
\hline por_G120D & $4 / 22(18.2)$ & $18 / 22(81.8)$ & $3 / 22(13.6)$ & $19 / 22(86.4)$ & 95.5 \\
\hline porB_G120K & $0 / 22$ & $22 / 22(100)$ & $0 / 22$ & $22 / 22(100)$ & 100 \\
\hline porB_A121G & $2 / 22(9.1)$ & $20 / 22(90.1)$ & $2 / 22(9.1)$ & $20 / 22(90.1)$ & 100 \\
\hline porB_A121D & $8 / 22(36.4)$ & $14 / 22(63.6)$ & $6 / 22(27.3)$ & $16 / 22(72.7)$ & 90.9 \\
\hline mtrR_G45D & $0 / 22$ & $22 / 22(100)$ & $0 / 22$ & $22 / 22(100)$ & 100 \\
\hline rpsJ_V57M & $22 / 22(100)$ & $0 / 22$ & $21 / 22(95.5)$ & $1 / 22(4.5)$ & 95.5 \\
\hline gyrA_S91F & $18 / 22(81.8)$ & $4 / 22(18.2)$ & $18 / 22(81.8)$ & $4 / 22(18.2)$ & 100 \\
\hline gyrA_D95A & $6 / 22(27.3)$ & $16 / 22(72.7)$ & $5 / 22(22.7)$ & $17 / 22(77.3)$ & 95.5 \\
\hline gyrA_D95G & $3 / 22(13.6)$ & $19 / 22(86.4)$ & $3 / 22(13.6)$ & $19 / 22(86.4)$ & 100 \\
\hline parC_S87R & $0 / 22$ & $22 / 22(100)$ & $0 / 22$ & $22 / 22(100)$ & 100 \\
\hline parC_S88P & $0 / 22$ & $22 / 22(100)$ & $0 / 22$ & $22 / 22(100)$ & 100 \\
\hline 16S_C1192U* & $0 / 22$ & $22 / 22(100)$ & $0 / 22$ & $22 / 22(100)$ & 100 \\
\hline penA_G545S* & $0 / 22$ & $22 / 22(100)$ & $0 / 22$ & $22 / 22(100)$ & 100 \\
\hline penA_mosaic* & $0 / 22$ & $22 / 22(100)$ & $0 / 22$ & $22 / 22(100)$ & 100 \\
\hline
\end{tabular}

from isolates and paired clinical specimens showed $>90 \%$ concordance, which indicates the potential to identify AMR targets directly from N. gonorrhoeae-positive vaginal/urethral specimens.

While multiple commercially available NAATs are available to diagnose STIs, this study used real-time PCR to detect the species-specific porA pseudogene that identifies $N$. gonorrhoeae. The concordance was $100 \%$ when compared with WGS. Other studies have also reported excellent clinical performance of porA [12, 27, 30, 48-50]. There have been reports of porA mutants which result in a false-negative for identification [51]; however, this was not detected in our isolates. This cost-effective real-time assay, as a diagnostic marker for reliable identification, can be introduced in a clinic management algorithm, and a result can be obtained within two hours compared to culture, which can take up to 48 hours for isolation of $N$. gonorrhoeae.

The prevalence of the $b l a_{T E M}$ (high-level resistance) in our study was $79 \%$. This is similar to a recent study from Africa, which reported a prevalence of $72 \%$ [52]. Resistance in isolates with intermediate MICs was chromosomally mediated. Modelling analysis identified tetM and rpsI_V57M to be excellent predictors of resistance to tetracycline [53]. All isolates in this study were resistant to tetracycline. The prevalence of the tet $M$ plasmid (high-level resistance) was $95 \%$. This is consistent with that of Kenya and South Africa reported recently as $86 \%$ and $92 \%$, respectively $[52,54]$. rpsJ_V57M (lower-level resistance) was also detected in all isolates resistant to tetracycline. This is similar to that reported in Vietnam [55], but higher compared to that found in Johannesburg, South Africa (70\%) [56], Brazil (39\%) [57], and Ukraine (67\%) [58].

Resistance to ciprofloxacin was detected in $82 \%$ of isolates, and gyrA_S91F SNP was detected in all resistant isolates. Other studies have also shown that this SNP is an excellent target for ciprofloxacin resistance detection $[55,56]$. The only available commercial assay for resistance detection, SpeeDX, has been developed based on this mutation.
All isolates from our patient population were susceptible to spectinomycin, and using HRM, we detected the resistance-associated SNP C1192U in the control strain. Spectinomycin resistance is exceedingly rare globally [59], and based on local resistance data, it is an effective treatment for genital and anorectal gonococcal infection [60]. A combination of ceftriaxone and spectinomycin is currently used in Japan [11]. South Korea also effectively treats gonorrhoea with spectinomycin, and resistance has not been reported since 1993 [61]. It is reassuring to know that we have the option to preserve the effectiveness of azithromycin and cefixime/ceftriaxone for use in gonorrhoeal infection by using spectinomycin as first-line therapy.

A limitation of this study is the small sample size, which has resulted in the low performance characteristics of some of the SNP targets when comparing HRM and WGS data. To better determine the performance, further evaluation of these drug targets is required on a much larger sample set from clinical specimens collected from hospitals and clinics across the different provinces in South Africa. porA mutants that result in false-negative identification for $N$. gonorrhoeae have been found in other studies [51], and in our subsequent studies, a combination of porA and opa will be used for identification. Also, the principal gene for azithromycin resistance, 23S rRNA, was not included in this HRM study and will be included in subsequent studies. However, from WGS data analysis, none of the isolates had mutations present in their $23 \mathrm{~S}$ rRNA genes. At present, due to unknown and novel mechanisms of resistance, genotypic AMR prediction cannot completely replace phenotypic AMR; therefore, continued and updated surveillance of local isolates is needed to identify mutations associated with resistance so that local in-house assays can be updated accordingly.

Cost-effective molecular diagnostic tools are required for rapid AMR detection, especially in low-middle-income countries. Using a diagnostic tool such as HRM, which is affordable and where multiple targets can be run in a single analysis, is an option for use in antimicrobial stewardship, 
rather than the currently used syndromic management approach. However, a functioning laboratory and trained personnel are required. We show that, in our local setting, the porA pseudogene can be used reliably to detect $N$. gonorrhoeae, bla $a_{\text {TEM }}$ can be used to detect HLR to penicillin, tet $M$ and rpsJ can be used to detect resistance to tetracycline, and gyrA_S91F can be used to detect resistance to ciprofloxacin.

\section{Data Availability}

Whole-genome sequence data are available in DDBJ/ENA/ GenBank with the BioProject number PRJNA681740.

\section{Disclosure}

The funders had no role in study design, data collection and interpretation, or the decision to submit for publication.

\section{Conflicts of Interest}

The authors have no conflicts of interest.

\section{Acknowledgments}

This study was funded by the DST-NRF Centre of Excellence (CoE) in HIV Prevention grant and the National Health Laboratory Service Research Trust grant. The authors acknowledge Prof. P. Moodley for donating a subset of isolates, V. Maseko, and the staff at the National Health Laboratory Service Microbiology Department (Durban).

\section{References}

[1] WHO, WHO factsheet 2019, WHO, Geneva, Switzerland, 2019, https://www.who.int/news-room/fact-sheets/detail/ sexually-transmitted-infections-(stis).

[2] W. H. Organization, "Global health sector strategy on sexually transmitted infections, 2016-2021,” WHO, Geneva, Switzerland, 2016.

[3] G. S. Hall, Bailey \& Scott's Diagnostic Microbiology, American Society for Clinical Pathology, Chicago, IL, USA, 13th edition, 2015.

[4] J. Serra-Pladevall, E. Caballero, G. Roig, R. Juvé, M. J. Barbera, and A. Andreu, "Comparison between conventional culture and NAATs for the microbiological diagnosis in gonococcal infection," Diagnostic Microbiology and Infectious Disease, vol. 83, no. 4, pp. 341-343, 2015.

[5] C. M. Wind, H. J. C. de Vries, M. F. Schim van der Loeff, M. Unemo, and A. P. van Dam, "Successful combination of nucleic acid amplification test diagnostics and targeted deferred Neisseria gonorrhoeae culture," Journal of Clinical Microbiology, vol. 53, no. 6, pp. 1884-1890, 2015.

[6] M. Tsadik, Y. Berhane, A. Worku, and W. Terefe, "The magnitude of, and factors associated with, loss to follow-up among patients treated for sexually transmitted infections: a multilevel analysis," BMJ Open, vol. 7, no. 7, p. e016864, 2017.

[7] C. Leeyaphan, S. Jiamton, P. Chanyachailert, T. Surawan, and V. Omcharoen, "Treatment outcomes and loss to follow-up rate of male patients with gonococcal and nongonococcal urethritis who attended the sexually transmitted disease clinic: an 8-year retrospective study," Indian Journal of Sexually Transmitted Diseases and AIDS, vol. 38, no. 1, pp. 37-42, 2017.

[8] S. C. Sonkar, K. Wasnik, A. Kumar, P. Mittal, and D. Saluja, "Comparative analysis of syndromic and PCR-based diagnostic assay reveals misdiagnosis/overtreatment for trichomoniasis based on subjective judgment in symptomatic patients," Infectious diseases of poverty, vol. 5, no. 1, pp. 42-10, 2016.

[9] S. C. Sonkar, K. Wasnik, A. Kumar et al., "Evaluating the utility of syndromic case management for three sexually transmitted infections in women visiting hospitals in Delhi, India," Scientific Reports, vol. 7, no. 1, pp. 1465-1469, 2017.

[10] B. Moges, G. Yismaw, A. Kassu et al., "Sexually transmitted infections based on the syndromic approach in Gondar town, northwest Ethiopia: a retrospective study," BMC Public Health, vol. 13, no. 1, pp. 143-145, 2013.

[11] M. Unemo and W. M. Shafer, "Antimicrobial resistance in Neisseria gonorrhoeae in the 21st century: past, evolution, and future," Clinical Microbiology Reviews, vol. 27, no. 3, pp. 587-613, 2014.

[12] S. Balashov, E. Mordechai, M. E. Adelson, and S. E. Gygax, "Multiplex bead suspension array for screening Neisseria gonorrhoeae antibiotic resistance genetic determinants in noncultured clinical samples," Journal of Molecular Diagnostics, vol. 15, no. 1, pp. 116-129, 2013.

[13] M. Christodoulides, Neisseria Gonorrhoeae: Methods and Protocols, Springer, Berlin, Germany, 2019.

[14] W. L. Veal, R. A. Nicholas, and W. M. Shafer, "Overexpression of the MtrC-MtrD-MtrE efflux pump due to an mtrR mutation is required for chromosomally mediated penicillin resistance in Neisseria gonorrhoeae," Journal of Bacteriology, vol. 184, no. 20, pp. 5619-5624, 2002.

[15] P. A. Ropp, M. Hu, M. Olesky, and R. A. Nicholas, "Mutations in ponA, the gene encoding penicillin-binding protein 1 , and a novel locus, penC, are required for high-level chromosomally mediated penicillin resistance in Neisseria gonorrhoeae," Antimicrobial Agents and Chemotherapy, vol. 46, no. 3, pp. 769-777, 2002.

[16] M. Unemo, D. Golparian, and D. W. Eyre, Antimicrobial Resistance in Neisseria Gonorrhoeae and Treatment of Gonorrhea. Neisseria gonorrhoeae, Springer, Berlin, Germany, 2019.

[17] X.-Z. Li, C. A. Elkins, and H. I. Zgurskaya, Efflux-mediated Antimicrobial Resistance in Bacteria: Mechanisms, Regulation and Clinical Implications, Springer, Berlin, Germany, 2016.

[18] M. Unemo, H. S. Seifert, E. W. Hook, S. Hawkes, F. Ndowa, and J.-A. R. Dillon, Gonorrhoea. Nature Reviews Disease Primers.vol. 5, no. 1, pp. 1-23, 2019.

[19] V. G. Allen, D. J. Farrell, A. Rebbapragada et al., "Molecular analysis of antimicrobial resistance mechanisms inNeisseria gonorrhoeaeIsolates from ontario, Canada," Antimicrobial Agents and Chemotherapy, vol. 55, no. 2, pp. 703-712, 2011.

[20] V. Donà, N. Low, D. Golparian, and M. Unemo, "Recent advances in the development and use of molecular tests to predict antimicrobial resistance in Neisseria gonorrhoeae," Expert Review of Molecular Diagnostics, vol. 17, no. 9, pp. 845-859, 2017.

[21] C. Ferreyra, J. Osborn, F. Moussy et al., "Developing target product profiles for Neisseria gonorrhoeae diagnostics in the context of antimicrobial resistance: an expert consensus," PloS one, vol. 15, no. 9, p. e0237424, 2020.

[22] A. R. Tuite, T. L. Gift, H. W. Chesson, K. Hsu, J. A. Salomon, and Y. H. Grad, "Impact of rapid susceptibility testing and antibiotic selection strategy on the emergence and spread of 
antibiotic resistance in gonorrhea," The Journal of Infectious Diseases, vol. 216, no. 9, pp. 1141-1149, 2017.

[23] C. A. Gaydos and J. H. Melendez, "Point-by-point progress: gonorrhea point of care tests," Expert Review of Molecular Diagnostics, vol. 20, no. 8, pp. 803-813, 2020.

[24] N. Garrett, A. Mtshali, F. Osman, L. Masson, L. R. McKinnon, and R. Singh, "Impact of point-of-care testing and treatment of sexually transmitted infections and bacterial vaginosis on genital tract inflammatory cytokines in a cohort of young south african women," Sexually Transmitted Infections, vol. 97, 2021.

[25] S. Bustin, "Real-time PCR," Encyclopedia of diagnostic genomics and proteomics, vol. 10, pp. 1117-1125, 2005.

[26] K. Tzimoula, E. Maria, D. Eudoxia, G. Georgia, M. Angeliki, and M. Nikolaos, "Detection of the tetM resistance determinant among phenotypically sensitive Ureaplasma species by a novel real-time PCR method," Diagnostic Microbiology and Infectious Disease, vol. 81, no. 2, pp. 85-88, 2015.

[27] M. Unemo, O. Norlen, and H. Fredlund, "The porA pseudogene of Neisseria gonorrhoeae- low level of genetic polymorphism and a few, mainly identical, inactivating mutations," Apmis, vol. 113, no. 6, pp. 410-419, 2005.

[28] D. M. WhileY, T. P. Anderson, K. Barratt et al., "Evidence that the gonococcal porA pseudogene is present in a broad range of Neisseria gonorrhoeae strains; suitability as a diagnostic target," Pathology, vol. 38, no. 5, pp. 445-448, 2006.

[29] S. Cotton, M. P. McHugh, M. Etherson, J. Shepherd, and K. E. Templeton, "Evaluation of the molecular detection of ciprofloxacin resistance in Neisseria gonorrhoeae by the ResistancePlus GC assay (SpeeDx)," Diagnostic Microbiology and Infectious Disease, vol. 99, no. 4, p. 115262, 2021.

[30] V. Dona, S. Kasraian, A. Lupo, Y. N. Guilarte, C. Hauser, and H. Furrer, "A multiplex real-time PCR with high resolution melting analysis for the characterization of antimicrobial resistance in Neisseria gonorrhoeae," Journal of Clinical Microbiology, vol. 54, 2016.

[31] S. O. Hjelmevoll, M. E. Olsen, J. U. E. Sollid, H. Haaheim, M. Unemo, and V. Skogen, "A fast real-time polymerase chain reaction method for sensitive and specific detection of the Neisseria gonorrhoeae porA pseudogene," Journal of Molecular Diagnostics, vol. 8, no. 5, pp. 574-581, 2006.

[32] L. B. Harrison and N. D. Hanson, "High-resolution melting analysis for rapid detection of sequence type 131 Escherichia coli," Antimicrobial Agents and Chemotherapy, vol. 61, no. 6, pp. e00265-17, 2017.

[33] B. J. Bricker, "Past, present and future of molecular technology applications for the epidemiology of bacterial diseases," Journal of Analytical \& Bioanalytical Techniques, vol. 2011, 2013.

[34] J. Twin, J. S. Jensen, C. S. Bradshaw et al., "Transmission and selection of macrolide resistant Mycoplasma genitalium infections detected by rapid high resolution melt analysis," PLoS One, vol. 7, no. 4, p. e35593, 2012.

[35] L.-T. Allan-Blitz, O. L. Ellis, R. Wee et al., "Improved determination of Neisseria gonorrhoeae gyrase A genotype results in clinical specimens," Journal of Antimicrobial Chemotherapy, vol. 74, no. 10, pp. 2913-2915, 2019.

[36] L. Xiu, C. Zhang, Y. Li, F. Wang, and J. Peng, "High-resolution melting analysis for rapid detection of the internationally spreading ceftriaxone-resistant Neisseria gonorrhoeae FC428 clone," Journal of Antimicrobial Chemotherapy, vol. 75, no. 1, pp. 106-109, 2020.

[37] N. J. Garrett, F. Osman, B. Maharaj et al., "Beyond syndromic management: opportunities for diagnosis-based treatment of sexually transmitted infections in low- and middle-income countries," PLoS One, vol. 13, no. 4, p. e0196209, 2018.

[38] M. Unemo, D. Golparian, L. Sánchez-Busó et al., “The novel 2016 WHONeisseria gonorrhoeaereference strains for global quality assurance of laboratory investigations: phenotypic, genetic and reference genome characterization," Journal of Antimicrobial Chemotherapy, vol. 71, no. 11, pp. 3096-3108, 2016.

[39] C.-H. Liao, C.-C. Lai, M.-S. Hsu et al., "Antimicrobial susceptibility of Neisseria gonorrhoeae isolates determined by the agar dilution, disk diffusion and Etest methods: comparison of results using GC agar and chocolate agar," International Journal of Antimicrobial Agents, vol. 35, no. 5, pp. 457-460, 2010.

[40] CLSI, "Methods for dilution antimicrobial susceptibility tests for bacteria that grow aerobically," Approved Standard-Ninth Edition, vol. 32, no. 2, 2012.

[41] The European Committee on Antimicrobial Susceptibility Testing, Breakpoint Tables for Interpretation of MICs and Zone Diameters, Sweden, 2017, http://www.eucast.org.

[42] F. Krueger: Trim Galore https://github.com/FelixKrueger/ TrimGalore.

[43] A. Bankevich, S. Nurk, D. Antipov et al., "SPAdes: a new genome assembly algorithm and its applications to single-cell sequencing," Journal of Computational Biology, vol. 19, no. 5, pp. 455-477, 2012.

[44] T. Seemann: Shovill https://github.com/tseemann/shovill.

[45] E. Zankari, R. Allesøe, K. G. Joensen, L. M. Cavaco, O. Lund, and F. M. Aarestrup, "PointFinder: a novel web tool for WGSbased detection of antimicrobial resistance associated with chromosomal point mutations in bacterial pathogens," Journal of Antimicrobial Chemotherapy, vol. 72, no. 10, pp. 2764-2768, 2017.

[46] L. Sánchez-Busó, C. A. Yeats, B. Taylor, R. J. Goater, A. Underwood, K. Abudahab et al., "A community-driven resource for genomic epidemiology and antimicrobial resistance prediction of Neisseria gonorrhoeae at Pathogenwatch," Genome Medicine, vol. 13, no. 1, pp. 1-22, 2021.

[47] F. Sievers, A. Wilm, D. Dineen et al., "Fast, scalable generation of high-quality protein multiple sequence alignments using Clustal Omega," Molecular Systems Biology, vol. 7, no. 1, p. 539, 2011.

[48] S. W. Peterson, I. Martin, W. Demczuk et al., "Molecular assay for detection of genetic markers associated with decreased susceptibility to cephalosporins in Neisseria gonorrhoeae," Journal of Clinical Microbiology, vol. 53, no. 7, pp. 2042-2048, 2015.

[49] M. L. Liu, Y. Xia, X. Z. Wu, J. Q. Huang, and X. G. Guo, "Loop-mediated isothermal amplification of Neisseria gonorrhoeae porA pseudogene: a rapid and reliable method to detect gonorrhea," AMB Express, vol. 7, no. 1, pp. 48-57, 2017.

[50] S. O. Hjelmevoll, D. Golparian, L. Dedi et al., "Phenotypic and genotypic properties of Neisseria gonorrhoeae isolates in Norway in 2009: antimicrobial resistance warrants an immediate change in national management guidelines," European Journal of Clinical Microbiology \& Infectious Diseases, vol. 31, no. 6, pp. 1181-1186, 2012.

[51] C. A. Ison, D. Golparian, P. Saunders, S. Chisholm, and M. Unemo, "Evolution ofNeisseria gonorrhoeaeis a continuing challenge for molecular detection of gonorrhoea: false negative gonococcalporAmutants are spreading internationally," Sexually Transmitted Infections, vol. 89, no. 3, pp. 197-201, 2013.

[52] M. W. Kivata, M. Mbuchi, F. Eyase, W. D. Bulimo, C. K. Kyanya, and V. Oundo, "Plasmid mediated penicillin 
and tetracycline resistance among Neisseria gonorrhoeae isolates from Kenya," BMC Infectious Diseases, vol. 20, no. 1, pp. 703-711, 2020.

[53] W. Demczuk, I. Martin, P. Sawatzky, V. Allen, B. Lefebvre, and L. Hoang, "Equations to predict antimicrobial MICs in Neisseria gonorrhoeae using molecular antimicrobial resistance determinants," Antimicrobial Agents and Chemother$a p y$, vol. 64, no. 3, 2020.

[54] S. Rambaran, K. Naidoo, N. Dookie, P. Moodley, and A. W. Sturm, "Resistance profile of Neisseria gonorrhoeae in KwaZulu-Natal, South Africa questioning the effect of the currently advocated dual therapy," Sexually Transmitted Diseases, vol. 46, no. 4, pp. 266-270, 2019.

[55] P. T. Lan, D. Golparian, J. Ringlander, L. Van Hung, N. Van Thuong, and M. Unemo, "Genomic analysis and antimicrobial resistance of Neisseria gonorrhoeae isolates from Vietnam in 2011 and 2015-16," Journal of Antimicrobial Chemotherapy, vol. 75, no. 6, pp. 1432-1438, 2020.

[56] L. D. Maduna, M. M. Kock, B. M. J. W. Van Der Veer et al., "Antimicrobial resistance of Neisseria gonorrhoeae isolates from high-risk men in Johannesburg, South Africa," Antimicrobial Agents and Chemotherapy, vol. 64, no. 11, 2020.

[57] A. P. Rd Costa-Lourenço, A. J. Abrams, K. T. B. Dos Santos, I. C. V. Argentino, T. Coelho-Souza, and M. C. A. Caniné, "Phylogeny and antimicrobial resistance in Neisseria gonorrhoeae isolates from Rio de Janeiro, Brazil," Infection, Genetics and Evolution, vol. 58, pp. 157-163, 2018.

[58] I. Boiko, D. Golparian, S. Jacobsson et al., "Genomic epidemiology and antimicrobial resistance determinants of Neisseria gonorrhoeae isolates from Ukraine, 2013-2018," Apmis, vol. 128 , no. 7 , pp. $465-475,2020$.

[59] M. Unemo, D. Golparian, V. Skogen et al., "Neisseria gonorrhoeae strain with high-level resistance to spectinomycin due to a novel resistance mechanism (mutated ribosomal protein S5) verified in Norway," Antimicrobial Agents and Chemotherapy, vol. 57, no. 2, pp. 1057-1061, 2013.

[60] W. H. Organization, WHO Guidelines for the Treatment of Neisseria Gonorrhoeae, World Health Organization, Berlin, Germany, 2016.

[61] M. Unemo, "Current and future antimicrobial treatment of gonorrhoea-the rapidly evolving Neisseria gonorrhoeae continues to challenge," BMC Infectious Diseases, vol. 15, no. 1, p. 364, 2015. 